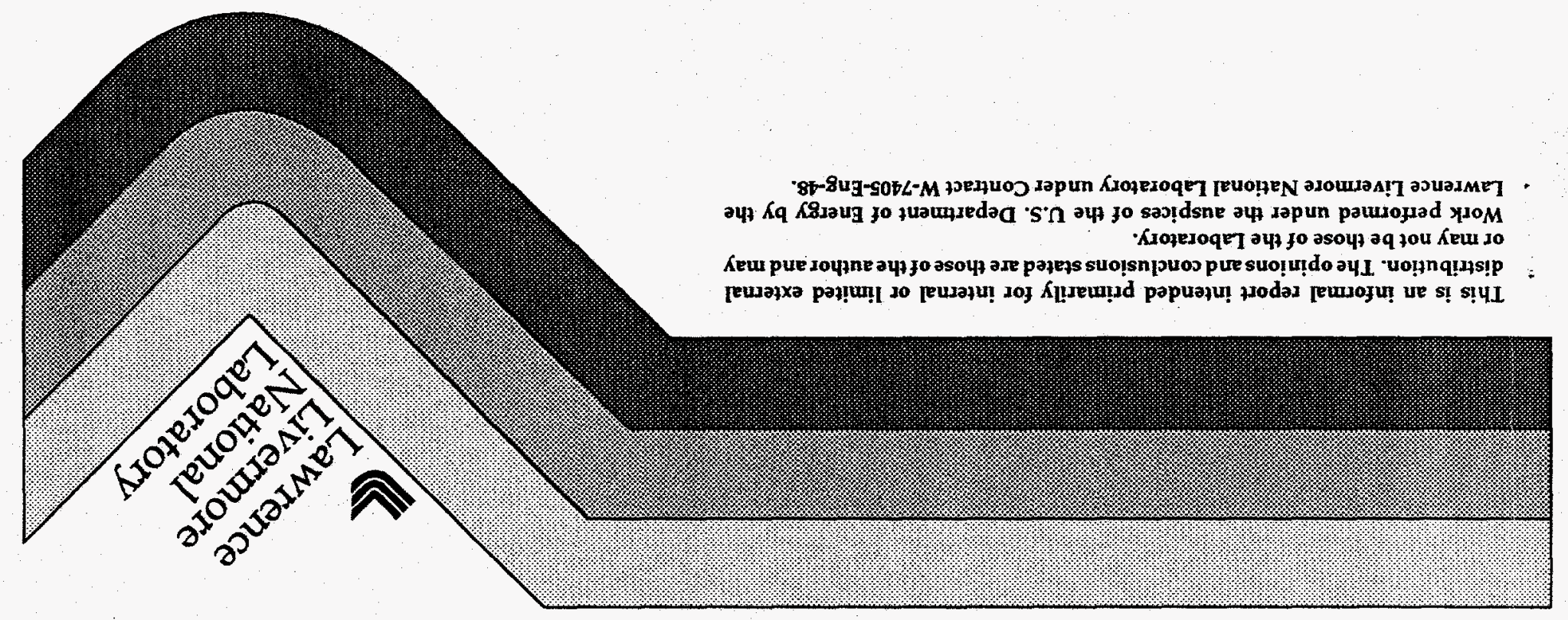

Ð66I 'L دәquәวәа

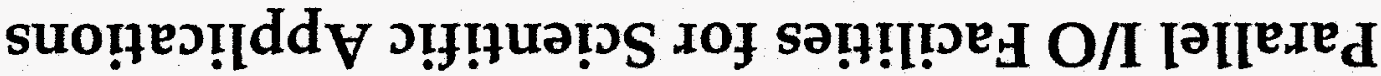

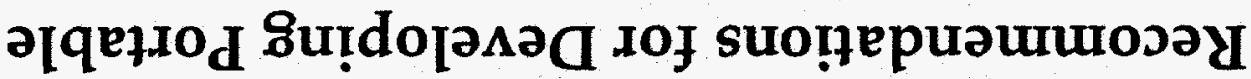




\section{DISCLAIMER}

Portions of this document may be illegible in electronic image products. Images are produced from the best available original document. 
This report was prepared as an account of work sponsored by an agency of the United States Government. Neither the United States Government nor any agency thereof, nor any of their employees, makes any warranty, express or implied, or assumes any legal liability or responsibility for the accuracy, completeness, or usefulness of any information, apparatus, product, or process disclosed, or represents that its use would not infringe privately owned rights. Reference herein to any specific commercial product, process, or service by trade name, trademark, manufacturer, or otherwise does not necessarily constitute or imply its endorsement, recommendation, or favoring by the United States Government or any agency thereof. The views and opinions of authors expressed herein do not necessarily state or reflect those of the United States Government or any agency thereof.

\title{
Recommendations for Developing Portable Parallel I/O Facilities for Scientific Applications
}

\author{
Robert K. Yates \\ Lawrence Livermore National Laboratory
}

December 1, 1994

\begin{abstract}
We outline a plan to develop portable parallel I/O facilities for scientific applications on parallel computers. We recommend that efforts be focussed on three areas: a new standard I/O library for message passing environments, efficient parallel I/O implementations for data parallel programming in Fortran 90 and High Performance Fortran (which may require new parallel I/O commands), and the promotion of high performance parallel I/O facilities in high-level scientific toolkits. In the next year we will concentrate primarily on the first area, designing and implementing a portable parallel I/O library to work with the new MPI standard Message Passing Interface.

Work performed under the auspices of the U.S. Department of Energy by Lawrence Livermore National Laboratory under contract number W-7405-Eng-48. This document was prepared as an account of work sponsored by an agency of the United States Government. Neither the United States Government nor the University of California nor any of their employees, makes any warranty, expressed or implied, or assumes any legal liability or responsibility for the accuracy, completeness, or usefulness of any information, apparatus, product, or process disclosed, or represents that its use would not infringe privately owned rights. Reference herein to any specific commercial products, process, or service by trade name, trademark, manufacturer, or otherwise, does not necessarily constitute or imply its endorsement, recommendation, or favoring by the United States Government or the University of California. The views and opinions of authors expressed herein do not necessarily state or reflect those of the United States Government or the University of California, and shall not be used for advertising or product endorsement purposes.
\end{abstract}




\section{Introduction}

Developers of scientific programs designed to execute on massiveley parallel computers need new parallel $\mathrm{I} / \mathrm{O}$ facilities to handle the large amounts of data that can be generated and processed on those systems. To be most useful, the new I/O facilities must be portable, long-lived and capable of high performance. The best way to achieve these objectives in Fiscal Year 1995 is through cooperation in broad-based groups promoting the adoption of new $\mathrm{I} / \mathrm{O}$ standards in message passing and data parallel programming environments and in higher-level tool kits for scientific programming.

\section{Two main programming models}

One of the biggest obstacles in programming parallel and distributed computer systems has been the lack of a standard programming model. In sequential computing, for example, the Fortran 77 and C/Unix programming environments are ubiquitous, and programs can readily be ported from one machine to another with minimal effort. Moreover, programs that perform well on one sequential architecture will usually perform well on any other sequential architecture. In parallel computing the opposite situation holds: each parallel computer has a different programming model, and programs developed for one architecture will usually not work very well, if at all, on any other. Since new parallel computers will continue to be introduced very rapidly, the need for more general and long-lived programming environments with portable high performance parallel I/O facilities is apparent.

Fortunately, the situation is beginning to improve. Broad-based standardization efforts involving many users and vendors of parallel computers have resulted in new standards for the two currently dominant programming models. For message passing there is the new Message Passing Interface standard (MPI) [4]. For the data parallel model there is the Fortran 90 language (F90) [1] and the less mature, more radical High Performance Fortran (HPF) [3]. Both MPI and F90 will be widely supported by parallel computer vendors, and HPF will at least be influential.

Neither MPI, F90 nor HPF addresses the problem of I/O. Fortran 90 inherits the sequential I/O facilities of Fortran 77 unchanged; so far, these have proved to be inadequate for high-performance parallel 
I/O on massively parallel architectures. The HPF forum considered several proposals to incorporate parallel I/O facilities, but all were rejected; the subject may be reconsidered in future HPF discussions. Discussion of parallel I/O facilities as part of or supplementary to the MPI standard has begun. To advance the interests of scientific application developers we recommend active participation and advocacy in discussions of $\mathrm{I} / \mathrm{O}$ facilities for MPI and HPF.

\section{Parallel I/O with message passing}

Message passing is currently the most popular technology for programming parallel and distributed computer systems, but it needs a standard library of parallel $\mathrm{I} / \mathrm{O}$ commands.

\subsection{Message passing: background}

Message passing systems such as Express, PVM, p4, and PARMACS have been available for years. The unnecessary proliferation of systems has hindered program portability.

Recently, an open committee of people from the computer industry, academia and government laboratories have defined a new message passing standard named MPI (for "Message Passing Interface"). The objective of the MPI forum was to develop a widely-used core library of commands for writing message-passing programs by establishing a practical, portable, efficient and flexible standard. It is expected that all major manufacturers of massively parallel computers will offer implementations of MPI. ${ }^{1}$ There is a good chance that MPI may supplant other systems to become the most commonly used message passing environment for scientific parallel programming, with high levels of vendor support, portability and performance.

Starting in November 1994 the MPI forum began to discuss possible changes and additions to the standard, including the possible inclusion of parallel $\mathrm{I} / \mathrm{O}$ commands. A preliminary proposal to extend the MPI standard to include I/O has already been circulated [2].

\footnotetext{
${ }^{1}$ For example, at Lawrence Livermore National Laboratory there is already an early version of MPI (developed at Argonne National Laboratory and Mississippi State University) running on the Meiko CS-2, and in 1995 there will be a native version of MPI for the Cray T3D.
} 
Because of the importance of message passing in scientific computing and the likely success of MPI as a widely-used standard, it is important that every reasonable effort be made to promote the introduction of parallel $\mathrm{I} / \mathrm{O}$ commands into MPI.

The standard I/O commands available in Fortran or $\mathrm{C}$ are inadequate because they cannot deal with multiple threads of control nor distributed data structures. New parallel I/O commands need to be added, allowing for very efficient implementations and fitting together well with MPI's syntax and semantics. The preliminary proposal [2] is an example of a promising approach.

\subsection{Message passing: recommendations}

Since message passing is the dominant programming model now and for at least the next few years, the most important goal for FY95 is the development and implementation of a portable parallel I/O library for message passing environments. The most promising approach is to push for the adoption of a standard set of I/O commands in MPI, working through the MPI forum. The steps to achieve this are:

1. Active participation in MPI forum discussions concerning $I / O$, beginning with meetings at the November Supercomputing Conference.

2. Encourage and help application developers to make their needs known to the MPI committee.

3. Work with industry, government labs, and academic researchers to implement an I/O library for MPI. At LLNL, special attention will be paid to implementations for the Meiko CS-2 and Cray T3D and, as far as possible, for networks of workstations.

4. Work with application developers to try out the new I/O facilities, evaluating them for performance and ease of use.

If the MPI committee fails to adopt I/O as part of the MPI standard, we will work to promote a stand-alone library that will accompany MPI as a separate library. If MPI fails to replace other message passing libraries, putting parallel I/O commands in MPI might well lead developers of competing parallel programming environments to include them also. 


\section{Parallel I/O with data parallelism}

The main alternative to message passing is data parallelism, as exemplified by Fortran 90 [1] and its extension, HPF ("High Performance Fortran") [3]. Both of these languages rely on standard sequential Fortran 77 I/O commands, which have so far proven inadequate to deal with distributed data on massively parallel processors.

\subsection{Data parallelism: background}

Data parallel programming differs from message passing in that there is a single (global) address space and a single thread of control. The single-threaded, loosely synchronous nature of the data parallel model makes it less flexible than message passing, and efficient execution depends more on the quality of the compiler, but proponents argue that program development is easier.

Fortran 90 and HPF are both extensions of Fortran 77, a standard sequential programming language. New features were added to operate on all the elements of specified subsections of arrays in parallel, and in the case of HPF to direct the partitioning of array data over distributed memory. However, neither Fortran 90 nor HPF extended the standard sequential Fortran 77 I/O facilities. Some proposals were made to add parallel I/O commands to HPF, but these have not been adopted because the standard Fortran 77 I/O commands were thought to be adequate.

It remains to be seen whether acceptable $\mathrm{I} / \mathrm{O}$ performance can be achieved by Fortran 90 and HPF programs using Fortran $77 \mathrm{I} / \mathrm{O}$, or whether new parallel I/O commands will need to be added. If new commands are needed they will differ from those suitable for message passing environments due to dissimilarities between the two programming models.

Experiments with new implementations of Fortran 90 and HPF must be conducted to determine whether the performance is satisfactory or not. If not, vendors and researchers must be pressed to solve the problem, either by improving the parallel implementations of standard $\mathrm{I} / \mathrm{O}$ inherited from Fortran 77 , or by introducing new standard commands for parallel $\mathrm{I} / \mathrm{O}$ in data parallel programs. 


\subsection{Data parallelism: recommendations}

As far as time permits, we will press for the development of highperformance $\mathrm{I} / \mathrm{O}$ facilities in Fortran 90 and more experimental systems like HPF. This will require determining whether or not present facilities are or can be made adequate, and if not, developing new standard I/O libraries for data parallel programming.

To accomplish this it will be necessary to monitor ongoing research and development of $\mathrm{I} / \mathrm{O}$ systems for F90 and HPF, making sure that application programmers' needs are being addressed, and if not, to propose new approaches. However, most effort during the coming year will focus on message passing, as described in section 3 .

\section{$5 \quad$ Parallel I/O in scientific tool kits}

The preceding discussion of $\mathrm{I} / \mathrm{O}$ in message passing and data parallel programming environments dealt implicitly with relatively low-level operations analogous to Fortran's commands to seek to a particular sequential location and to read or write blocks of data. Another implicit restriction was to simple data structures such as regular rectangular arrays, whereas irregular data structures like the complex meshes found in finite element codes are extremely important in practice. There is a great advantage to be gained by providing high-performance parallel I/O at a higher semantic level.

\subsection{Scientific tool kits: background}

NetCDF [6] and HDF [8], for example, are popular scientific data management packages that support machine-independent self-describing files for complex data structures. They perform numeric format conversions and data compression. They provide high-level utilities and operators to make it easier to store, access, analyze and display large, complex scientific data sets. Another sort of toolkit for scientific program development is exemplified by PETSc [5], which is a library of utilities offering ready-made implementations of high-level scientific algorithms.

Obviously, the utility of this sort of scientific data management tool would be much enhanced by the provision of high-level parallel I/O facilities. Some work in this area has been done at the University 
of Illinois [7], and there is some hope that HDF, at least, may benefit from this sometime in the future.

It is in the interests of scientific application developers to push for the development of scientific data management tools with fast, highlevel I/O facilities for parallel computers. Much more work will be needed. A dialog between users, designers, and implementors must be established to ensure that these tools will be efficient and easy to use.

\subsection{Scientific tool kits: background}

We will push for the inclusion of high-performance parallel I/O facilities in high-level scientific toolkit packages. Probably the only thing we can do this coming year is publicize the need to upgrade the $1 / O$ capabilities of existing packages, and try to facilitate dialogue between toolkit designers, I/O researchers, and potential users. Most effort will focus on message passing, as described in section 3 .

\section{Conclusion}

The best way to make portable high-performance I/O facilities available to scientific application developers is through cooperation with groups interested in developing widely used standards supported by industry. The advantage of widely accepted standards is greater portability and better performance through cooperation and competition.

We have identified three areas where improvement would have the most beneficial effects: the definition and implementation of new $1 / O$ commands for a widely-used message passing environment, the evaluation of I/O implementations in data parallel versions of Fortran with possible recommendations for new approaches, and the promotion of high performance parallel I/O facilities in high-level scientific toolkits.

In the coming year we will focus our efforts in the first area, designing and implementing a portable parallel I/O library for inclusion in the new MPI standard message passing interface. 


\section{References}

[1] Adams, Brainerd, Martin, Smith, and Wagener. The FORTRAN 90 Handbook. McGraw-Hill, 1990.

[2] Peter F. Corbett, Dror G. Feitelson, Yarsun Hsu, Jean-Pierre Prost, Marc Snir, Sam Fineberg, Bill Nitzberg, Bernard Traversat, and Parkson Wong. MPI-IO: A parallel file interface for MPI. Technical report, 1994. Available on WWW at http://lovelace.nas.nasa.gov/MPI-IO/mpi-io.html.

[3] High Performance Fortran Forum. High performance FORTRAN language specification, version 1.0. Technical report, Center for Research in Parallel Computation, Rice University, Houston, TX, January 1993. Tech. Rpt. CRPC-TR92225.

[4] Message Passing Interface Forum. MPI: A message-passing interface standard. Technical Report Computer Science Department Technical Report CS-94-230, University of Tennessee, Knoxville, TN, May 5 1994. To appear in the International Journal of Supercomputing Applications, Volume 8, Number 3/4, 1994.

[5] W. Gropp and B. Smith. Scalable, extensible, and portable numerical libraries. In Proc. of the Scalable Parallel Libraries Conf., pages 87-93, 1994.

[6] Harry L. Jenter and Richard P. Signell. NetCDF: A public-domain software solution to data access problems for numerical modelers. 1992.

[7] Kent E. Seamons and Marianne Winslett. An efficient abstract interface for multidimensional array I/O. In Proceedings of Supercomputing 94. IEEE Computer Society Press, 1994.

[8] Software Development Group. HDF: Hierarchical Data Format. Technical report,

National

Center for Supercomputing Applications. Available on WWW at http://www.ncsa.uiuc.edu/SDG/Software/Brochure/DSTSoftDesc.html\#HDF. 